\title{
Study on Influencing Factors of Sodium Modification of Bentonite
}

$$
\text { Xiaobing Man }{ }^{1,} \text {, } \text {, Xuan Zhang }{ }^{2, b} \text {, Bo Wang }{ }^{3, c}
$$

${ }^{1,2}$ School of Environmental Science and Engineering, Qilu University of Technology, Jinan 250353, China;

${ }^{3}$ Shan Dong Accreditation Center forEnvironmental Impact Assessment, Jinan 250353, China.

amxb0828@163.com, bahongjn@126.com, ceia001@126.com

Keywords: bentonite, sodium modification, characterization

\begin{abstract}
Bentonite, a new class of layered compounds, displayed a broad application prospects in catalyst supports. The influencing factors of sodium modification on bentonite were studied in this paper. The bentonite obtained the best charater after the $10 \%$ bentonite slurry was treated by $3 \%$ $\mathrm{Na}_{2} \mathrm{CO}_{3}$ at $60^{\circ} \mathrm{C}$ for $1.5 \mathrm{~h}$. It can be seen that the swellvalue increased from $19.7 \mathrm{ml} / \mathrm{g}$ to $86.2 \mathrm{ml} / \mathrm{g}$, the blue absorption power from $28.8 \mathrm{~g} / 100 \mathrm{~g}$ to $43.6 \mathrm{~g} / 100 \mathrm{~g}$ and the cationic exchange capacity from $0.384 \mathrm{mmol} / \mathrm{g}$ to $1.026 \mathrm{mmol} / \mathrm{g}$. The X-ray Diffraction showed the interlayer space changed from $1.4321 \mathrm{~nm}$ to $1.229 \mathrm{~nm}$. All the results meant that the Ca-bentonite had been modified into Na-bentonite and obtained good expansibility and dispersibility.
\end{abstract}

\section{Introduction}

With the progress of science and technology, the requirements of bentonite are getting higher and higher. In China, bentonite reserve ranked first in the world. But most of them was Ca-bentonite ${ }^{[1]}$. It contained some non-montmorillonit components such as quartz, feldspar, cristobalite and other impurities, resulting that further purification was needed for bentonite to make better use ${ }^{[2]}$. The Na-bentonite, having larger specific surface area, higher cationic exchange properties, better physical properties, had broader market prospects. Ca-Bentonite could be modified by ion exchange to prepare Na-bentonite ${ }^{[3]}$. The Na-bentonite has better water absorption and expansibility in an aqueous medium dispersion. And also its colloidal suspensions thixotropic and thermal stability were better than Ca-bentonite ${ }^{[4]}$. The application of Na-bentonite was more widely Ca-bentonite. The study on sodium modification of bentonite was of great significance for the preparation of Na-bentonite with high performance.

In this paper, the influencing factors of sodium modification were studied. The obtained Na-bentonite was characterized by X-ray diffraction (XRD) and scanning electronic microscopy (SEM).

\section{Experimental}

\subsection{Materials}

$\mathrm{Na}_{2} \mathrm{CO}_{3}, \mathrm{HCl}, \mathrm{Na}_{4} \mathrm{P}_{2} \mathrm{O}_{7}, \mathrm{MgO}$, Methylene blue, $\mathrm{NH}_{4} \mathrm{Cl}, \mathrm{NH}_{3} \cdot \mathrm{H}_{2} \mathrm{O}$, ethanol absolute, formalin, $\mathrm{CaCl}_{2}$ and phenolphthalein were supplied by Tianjin Kemiou Chemical Reagent Co. Ltd. All chemicals were of analytical reagent grade and used without further purification.

Bentonite was obtained commercially and was used without any further purification.

\subsection{Influencing factors of sodium modification}

The experiments for the influencing factors of sodium modification were as follows. Firstly, the bentonite was added into a certain amount of water and stirred for $2 \mathrm{hr}$ at $60^{\circ} \mathrm{C}$. Secondly, amount of $\mathrm{Na}_{2} \mathrm{CO}_{3}$ was added into the slurry in an Erlenmeyer flask and shaken for some hour at some temperature. Lastly, the slurry was filtered by filter paper after standing for 20 minutes and washed with distilled water and dried at $85^{\circ} \mathrm{C}$ for $16 \mathrm{hr}$. Finally, the dried solid was ground and sieved to obtain a fraction passing through 200 mash screen.

In this paper, the dosage of sodium agent, reaction time, slurry concentration and reaction temperature were studied. 


\subsection{Characterization}

The swellvalue, the blue absorption power and the cationic exchange capacity (CEC) were examined by GB/T 20973-2007, which was published on June 22, 2007 in China. The XRD was done in a 'Bruker D8 Advance' device, which operates with $\mathrm{Cu} \mathrm{K} \alpha$ radiation that ranged between $5^{\circ}$ and $40^{\circ}$. The SEM was done in a 'FEI Quanta 200' device, which delivered with a $50 \mathrm{~mm}$ (2 inch) motorized stage (X/Y travel), with a motorized z-range of $25 \mathrm{~mm}$.

\section{Result and Discussion}

\subsection{Effect of the dosage of sodium agent}

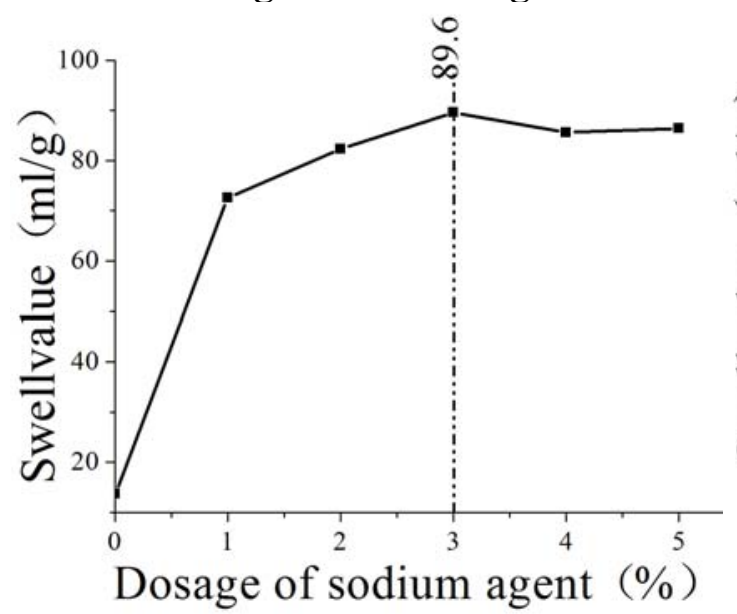

Fig.1. Effect dosage of sodium agent on the quality of Na-bentonite

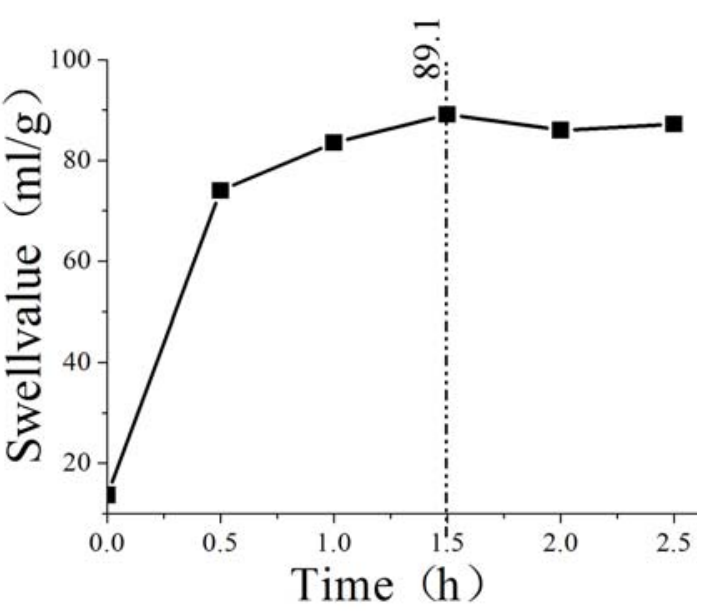

Fig.2.Effect of the reaction time on the quality of Na-bentonite

The influence of the dosage of sodium agent in sodium modification was illustrated in Fig.1. The swellvalue increased as the amount of $\mathrm{Na}_{2} \mathrm{CO}_{3}$ increased, when the dosage of sodium agent was less than $3 \%$. The reaction was driven by the difference of ions concentrations. Only if the concentration of $\mathrm{Na}^{+}$was greater than the amount of $\mathrm{Ca}^{2+}$ and $\mathrm{Mg}^{2+}$, could the sodium modification react completely. The swellvalue decreased slightly, when the dosage of sodium agent was more than 3\%. Because the equilibrium of ion exchange between $\mathrm{Ca}^{2+}$ and $\mathrm{Na}^{+}$was destroyed, the effect of more sodium agent on the sodium modification was poor. Therefore, the best dosage of $\mathrm{Na}_{2} \mathrm{CO}_{3}$ was determined to be $3 \%$.

\subsection{Effect of the reaction time}

Enough time was important to complete the sodium reaction. The swellvalue increased quickly, when the time was less than $0.5 \mathrm{hr}$ (Fig.2). It reached the highest value, when the time was $1.5 \mathrm{hr}$. When the slurry was treated for more than $1.5 \mathrm{hr}$, the swellvalue began to decrease. It could be seen $1.5 \mathrm{hr}$ was the best time for sodium modification. More time would destroy the equilibrium of ion exchange. In addition, the sample would become worse dispersibility and more difficult to dewater if the slurry was treated for a longer time.

\subsection{Effect of the slurry concentration}

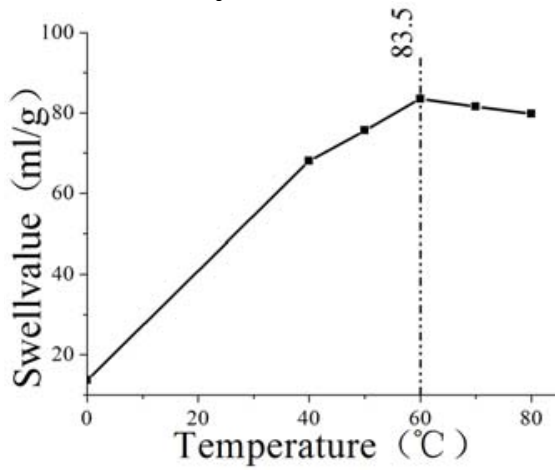

Fig.3. Effect of the slurry concentration on the swellvalue of Na-bentonite

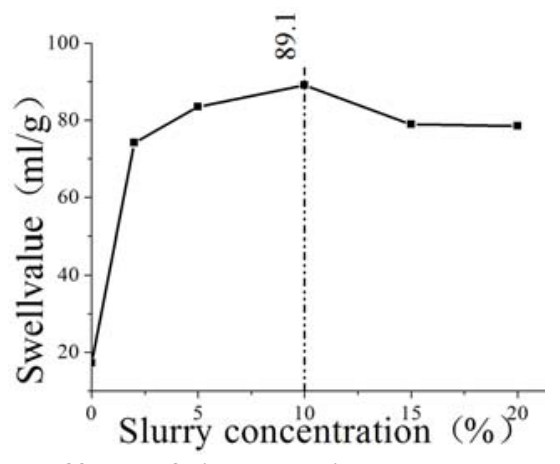

Fig.4. Effect of the reaction temperature on the swellvalue of $\mathrm{Na}$-bentonite 
The effect of slurry concentration on the swellvalue was shown in Fig.3. The highest swellvalue was obtained when the slurry concentration was $10 \%$. The low concentration would increase the load during the subsequent dehydration process. When the slurry concentration was more than $10 \%$, the liquidity of the slurry would become poor and it was hard for the slurry and ion $\mathrm{Na}^{+}$to contact effectively. In consequence, the most suitable slurry concentration was chosen to be $10 \%$.

\subsection{Effect of the reaction temperature}

The temperature had a vital affect on the ions' movements. The higher was the temperature, the active was the ion. If the temperature began to increase from a low temperature, the moving rate of the ions increased quickly and also the viscosity decreased rapidly. The equilibrium between $\mathrm{Ca}^{2+}$ and $\mathrm{Na}^{+}$required short time, helping the sodium modification to complete in a short time. It could be seen in Fig.4 that the swellvalue increased when the reaction temperature was lower than $60^{\circ} \mathrm{C}$. At the same time, the higher was the temperature, the greater the ionization of $\mathrm{Ca}^{2+}$ was. If the temperature was higher than $60{ }^{\circ} \mathrm{C}$, the ionization $\mathrm{Ca}^{2+}$ would become a key factor and the ion equilibrium between $\mathrm{Ca}^{2+}$ and $\mathrm{Na}^{+}$moved to $\mathrm{Na}^{+}$. More $\mathrm{Na}^{+}$desorbed from the bentonite, resulting in the decrease of the swellvalue. As a consequence, the best reaction temperature was determined to be $60^{\circ} \mathrm{C}$.

\subsection{Characterization}
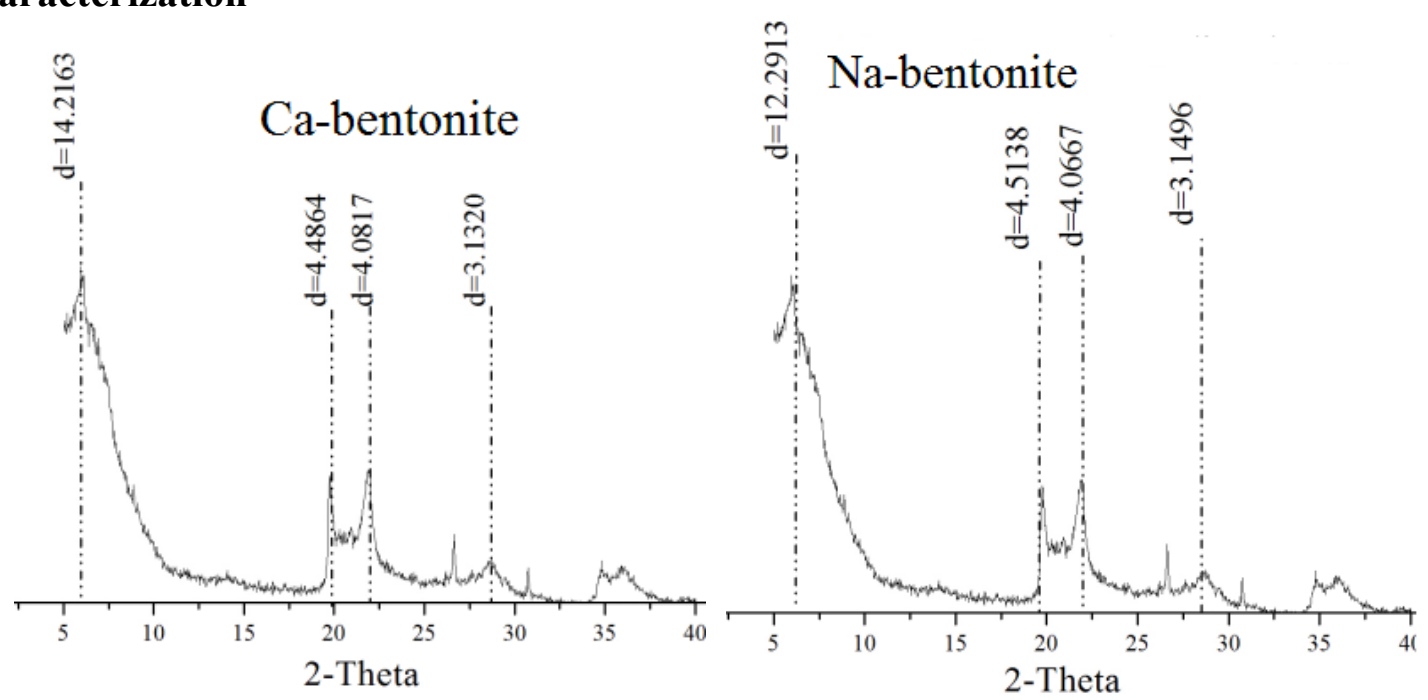

Fig.5. XRD patterns of Ca-bentonite and Na-bentonite

The raw bentonite was modified under the determined conditions. The character of Na-bentonite was shown in Table 1. The XRD (Fig.5) patterns shown that $\mathrm{d}_{(001)}$ had changed from $1.421 \mathrm{~nm}$ to $1.229 \mathrm{~nm}$, showing that Na-bentonite was obtained by the modification of the Ca-bentonite. Fig.6 was the SEM photos of bentonite. It could be seen that Na-bentonite had better expansibility and high dispersibility than Ca-bentonite. The particles were decomposed into thin wafers, showing an aggregate of petals and had obvious characteristics of layer and flake on the edge. Some part of the edge showed serration. Conclusion could be drawn that the bentonite obtained high purity and good performance after sodium modification. The content of montmorillonite increased from $65.1 \%$ to $98.6 \%$. 


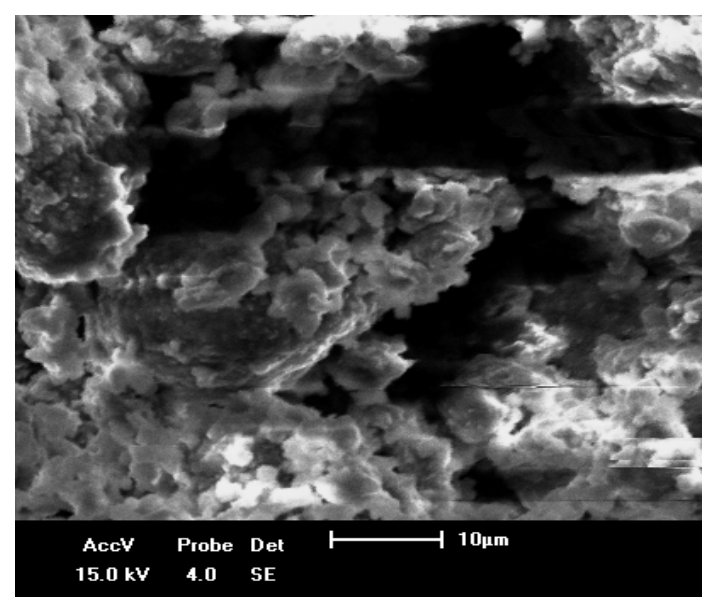

Ca-Bentonite

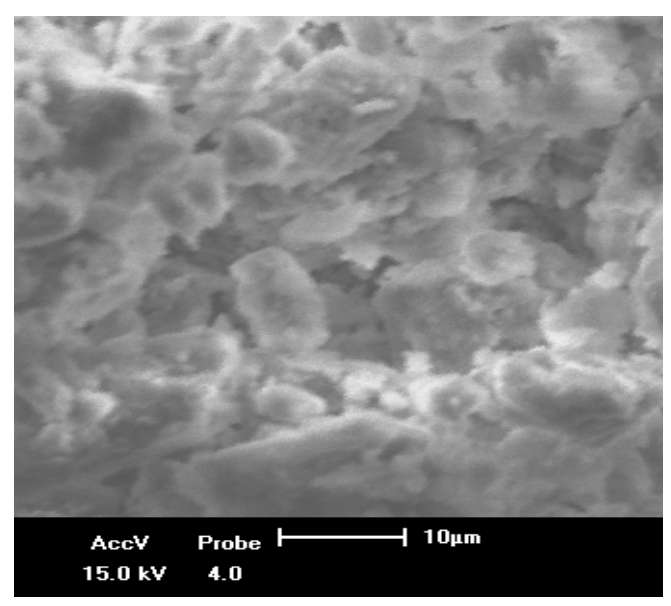

Na-bentonite

Fig.6. SEM photos of bentonite

Table.1 Character of bentonite

\begin{tabular}{ccc}
\hline Character & Ca-bentonite & Na-bentonite \\
\hline Swellvalue $(\mathrm{ml} / \mathrm{g})$ & 19.7 & 86.2 \\
Blue absorption power $(\mathrm{g} / 100 \mathrm{~g})$ & 28.8 & 43.6 \\
CEC $(\mathrm{mmol} / \mathrm{g})$ & 0.384 & 1.026 \\
\hline
\end{tabular}

\section{Conclusions}

Na-bentonite was obtained by the modification of commercial Ca-bentonite. $\mathrm{Na}_{2} \mathrm{CO}_{3}$ was chosen as the sodium agent and its dosage was determined to be $3 \%$. The $10 \%$ slurry was treated at $60^{\circ} \mathrm{C}$ for $1.5 \mathrm{~h}$, resulting that the swellvalue increased from $19.7 \mathrm{ml} / \mathrm{g}$ to $86.2 \mathrm{ml} / \mathrm{g}$, the blue absorption power from $28.8 \mathrm{~g} / 100 \mathrm{~g}$ to $43.6 \mathrm{~g} / 100 \mathrm{~g}$ and the CEC from $0.384 \mathrm{mmol} / \mathrm{g}$ to 1.026 $\mathrm{mmol} / \mathrm{g}$. The XRD showed the interlayer space changed from $1.4321 \mathrm{~nm}$ to $1.229 \mathrm{~nm}$. The SEM photos showed the Na-bentonite could obtain good expansibility and dispersibility. This experiment laid a good foundation for the preparation of pillaring bentonite.

\section{Acknowledgements}

This work was supported by the natural science foundation of Shandong province (Grant No. ZR2014JL012), Science and technology project of higher education of Shandong province (J13LDO2).

\section{References}

[1] Zhou Jiarong, Yi Facheng, Hou Li, et al. Study status on sodium modified method of Ca-bentonite. China Mining Magazine. Vol. 16(2007) No. 3, p.84-87.

[2] Yang Xiaojing, Mo Wei, Ma Shaojian, et al. Experimental study on sodium modification of Ca-bentonite from Guang xi. China. Non-Metallic Mining Industry Herald. Vol. 107(2013) No.6, p.16-19.

[3] Xu Xiaolong, Liu Lanjun. Research progress and application of sodium modified and inorganic pillared bentonite. Contemporary Chemical Industry. Vol. 42(2013) No.9, p.1261-1263.

[4] Wang Ge, Guo Haiying, Xu Guangliang, et al. Comparison method of purification and on Na-activation of bentonite. China Mining Magazine. Vol. 21(2012) No.2, p.85- 88. 